\title{
A Double-Blinded Randomized Study Investigating a Possible Anti-Inflammatory Effect of Saxagliptin versus Placebo as Add-On Therapy in Patients with Both Type 2 Diabetes And Stable Coronary Artery Disease
}

\author{
Ida Unhammer Njerve, ${ }^{1,2,3}$ Sissel Åkra, ${ }^{1,2}$ Thomas W. Weiss, ${ }^{4}$ Svein Solheim, ${ }^{1,2}$ \\ Reidun Øvstebø, ${ }^{5}$ Hans Christian D. Aass, ${ }^{5}$ Rune Byrkjeland, ${ }^{1,2,3}$ Harald Arnesen, ${ }^{1,2,3}$ and \\ Ingebjørg Seljeflot ${ }^{1,2,3}$ \\ ${ }^{1}$ Center for Clinical Heart Research, Department of Cardiology, Oslo University Hospital Ullevaal, Oslo, Norway \\ ${ }^{2}$ Center for Heart Failure Research, Oslo University Hospital Ullevaal, Oslo, Norway \\ ${ }^{3}$ Faculty of Medicine, University of Oslo, Oslo, Norway \\ ${ }^{4} 3 r d$ Medical Department for Cardiology, Wilhelminenspital, Vienna, Austria \\ ${ }^{5}$ The Blood Cell Research Group, Department of Medical Biochemistry, Oslo University Hospital Ullevaal, Oslo, Norway
}

Correspondence should be addressed to Ida Unhammer Njerve; i.u.njerve@medisin.uio.no

Received 24 November 2016; Revised 31 March 2017; Accepted 6 April 2017; Published 17 May 2017

Academic Editor: Julio Galvez

Copyright ( 2017 Ida Unhammer Njerve et al. This is an open access article distributed under the Creative Commons Attribution License, which permits unrestricted use, distribution, and reproduction in any medium, provided the original work is properly cited.

\begin{abstract}
Background. Promising results regarding potential anti-inflammatory and antiatherosclerotic effects of gliptins have been reported. Our aim was to investigate whether saxagliptin treatment modifies expression of inflammatory markers, primarily in peripheral blood mononuclear cells (PBMCs) and in circulating leukocytes in patients with stable coronary artery disease (CAD) and T2DM. Methods. Patients $(n=12)$ were randomized to saxagliptin $5 \mathrm{mg}$ daily or placebo for 3 months. Samples were taken at baseline and end of study in fasting state prior to intake of medications. PBMCs were isolated and cryopreserved at $-150^{\circ} \mathrm{C}$ until ex vivo exposed to $1 \mathrm{ng} / \mathrm{mL}$ of lipopolysaccharide (LPS) for 4 hours. Gene expression was performed with custom-designed TaqMan ${ }^{\circledR}$ Arrays and relative quantification by real-time PCR (RT-qPCR). Results. HbA1c was reduced in the saxagliptin-treated group compared to that in the change with placebo $(p=0.042)$. In unstimulated PBMCs and in circulating leukocytes, we observed a significant increase in IL-10 expression in the saxagliptin group $(p=0.043$, both), significantly different from that in the placebo $(p=0.009$ and $p=0.032$, resp.). No between group differences in changes were observed in any of the selected proinflammatory markers. Conclusion. In our small cohort of patients with combined T2DM and CAD, a possible anti-inflammatory effect of saxagliptin, observed in the present study by upregulation of IL-10 in leukocytes, needs to be confirmed in larger studies.
\end{abstract}

\section{Background}

Gliptins or dipeptidyl peptidase-4 (DPP-4) inhibitors are used as either monotherapy or, more frequently, as add-on therapy to other oral antidiabetic drugs in type 2 diabetes (T2DM) [1, 2]. They delay the inactivation of incretins glucagon-like peptide-1 (GLP-1) and glucose-dependent insulinotropic polypeptide (GIP) after meals, reducing postprandial glucose levels and also HbA1c levels [3].
DPP-4 is an enzyme with substrates other than the incretin hormones, including cytokines that might influence inflammation and atherosclerosis [4]. Animal studies have shown promising results regarding anti-inflammatory and antiatherosclerotic effects of gliptins in general [5-7]. Also, some clinical studies have been published showing a potential effect on atherosclerosis and inflammation in patients with T2DM using DPP-4 inhibitors as compared to that in patients using other antidiabetic drugs or placebo [8-11]. 
This has led to the hypothesis that DPP-4 inhibitors might have beneficial anti-inflammatory effects in addition to glucose-lowering effects.

The aim of our study was therefore to investigate whether the DPP-4 inhibitor saxagliptin modifies the expression of selected inflammatory biomarkers, primarily in isolated peripheral blood mononuclear cells (PBMCs) and in circulating leukocytes in patients with stable coronary artery disease (CAD) and T2DM. In addition, circulating levels of the markers were measured.

The selected markers were tumor necrosis factor- $\alpha$ (TNF- $\alpha$ ), interleukin-1 $\beta$ (IL- $1 \beta$ ), IL-18, monocyte chemoattractant protein-1 (MCP-1), fractalkine (CX3CL1) and its receptor CX3CR1, and the anti-inflammatory cytokine IL-10.

\section{Materials and Methods}

2.1. Patients and Study Design. Patients $(n=12)$ with both T2DM and stable CAD recruited from the Department of Cardiology, Oslo University Hospital Ullevaal, Oslo, Norway, from 2012-14, were randomized double blinded to placebo or saxagliptin ( $5 \mathrm{mg} /$ day) for 3 months. All study participants were adults, had angiographically verified stable CAD, and had a history of T2DM. Inclusion criteria were use of metformin and/or glimepiride for their T2DM and HbA1c levels $>6.5 \%$. Exclusion criteria for the study included allergy or hypersensitivity to any of the drug's components, heart failure NYHA class III and IV, severe liver failure, moderate or severe kidney failure, malignant disease, active infectious disease, acute coronary syndrome during the last three months, pregnancy, or breastfeeding.

All patients gave written, informed consent to participate in the study. The study was conducted in accordance with the Declaration of Helsinki and consistent with Good Clinical Practice, and the Regional Ethics Committee and the Norwegian Medicines Agency approved the protocol. ClinicalTrials .gov identifier: NCT01552018.

2.2. Sampling Procedures. Blood samples, including PAX gene blood RNA tubes (PreAnalytix Qiagen GmBH, Germany) and $\mathrm{BD}$ vacutainer $\mathrm{CPT}$ tubes for isolation of $\mathrm{PBMC}$ (Becton, Dickinson and Company, Franklin Lakes, USA), were drawn by standard venipuncture between 08.00 and $10.00 \mathrm{am}$ after an overnight fast and before intake of morning medication at inclusion and after 3-months of follow-up. Serum and plasma for determination of selected biomarkers were prepared by centrifugation within 1 hour at $2500 \times \mathrm{g}$ for $10 \mathrm{~min}$ and $4^{\circ} \mathrm{C}$ at $3000 \times \mathrm{g}$ for $20 \mathrm{~min}$, respectively. The samples were stored at $-80^{\circ} \mathrm{C}$ until analyses.

PBMCs were isolated and cryopreserved within 2 hours. In brief, cells were isolated by centrifugation at several steps, added 5\% FCS/RPMI, and counted for the distribution of monocytes and lymphocytes, recorded by flow cytometry using BD Accuri C6 (BD Biosciences, 2350 Qume Drive, San Jose, CA). Diluted DMSO/RPMI (20\%) was added before freezing at $-150^{\circ} \mathrm{C}$. Cells were further ex vivo exposed to lipopolysaccharide (LPS) with a final concentration of $1 \mathrm{ng} / \mathrm{mL}$ for 4 hours. Equal number of cells was used in all
TABle 1: Baseline characteristics according to the randomized groups. Median (25, 75 percentiles) or number is given.

\begin{tabular}{|c|c|c|}
\hline Characteristics & Saxagliptin & Placebo \\
\hline Age (years) & $68.5(63.0,73.0)$ & $64.0(58.5,67.5)$ \\
\hline Number (female) & $6(0)$ & $6(1)$ \\
\hline Years of diabetes & $7.8 \pm 4.4$ & $9.2 \pm 3.6$ \\
\hline Years of CAD & $6(1,15)$ & $2(1,7)$ \\
\hline Hypertension $(n)$ & 5 & 6 \\
\hline Previous AMI $(n)$ & 3 & 3 \\
\hline Current smoker $(n)$ & 2 & 1 \\
\hline $\mathrm{SBP}(\mathrm{mmHg})$ & $128(121,144)$ & $133(123,145)$ \\
\hline $\mathrm{DBP}(\mathrm{mmHg})$ & $75(68,79)$ & $74(69,80)$ \\
\hline Waist circumference $(\mathrm{cm})$ & $103(90,117)$ & $105(94,110)$ \\
\hline $\operatorname{BMI}\left(\mathrm{kg} / \mathrm{m}^{2}\right)$ & $28.1(25.1,32.3)$ & $28.6(24.7,29.0)$ \\
\hline \multicolumn{3}{|l|}{ Medication $(n)$} \\
\hline Glimepiride & 2 & 1 \\
\hline Metformin & 5 & 6 \\
\hline Platelet inhibitors & 4 & 5 \\
\hline ACE-inhibitors or ARBs & 4 & 6 \\
\hline Betablocker & 5 & 6 \\
\hline Statin & 6 & 6 \\
\hline Diuretic & 3 & 1 \\
\hline
\end{tabular}

AMI: acute myocardial infarction; SBP: systolic blood pressure; DBP: diastolic blood pressure; BMI: body mass index; ACE: angiotensin converting enzyme; ARB: angiotensin II receptor blocker.

experiments. The method is according to what is previously reported with a minor modification [12].

2.3. Laboratory Analyses. Fasting glucose, HbA1c, C-peptide, and insulin were determined by conventional laboratory methods. Glucose was analyzed by enzyme immunoassay (Cobas 8000, Roche Diagnostics, Basel, Switzerland), $\mathrm{HbA}_{1 \mathrm{c}}$ by turbidimetric inhibition immunoassay (Roche Diagnostics), insulin by DELFIA method (Perkin Elmer, Waltham, Massachusetts, USA), and C-peptide by electrochemiluminescence immunoassay (ECLIA) (Roche Diagnostics).

For gene expression analyses, total RNA was isolated from unstimulated and stimulated PBMCs by use of the RNeasy ${ }^{\circledR}$ Mini Kit (Qiagen) and from PAXgene tubes by use of a PAXGENE ${ }^{\circledR}$ Blood RNA Kit (PreAnalytix, Qiagen, $\mathrm{GmBH}$ ), with an extra cleaning step (RNeasy ${ }^{\circledR}$ MinElute ${ }^{\circledR}$ Cleanup Kit, Qiagen). RNA concentration (ng/ $\mu \mathrm{L})$ and quality was measured by the NanoDrop ${ }^{\mathrm{TM}} 1000$ Spectrophotometer (Thermo Scientific, Wilmington, Delaware, USA).

A predefined RNA concentration of $5 \mathrm{ng} / \mu \mathrm{L}$ was used for cDNA synthesis performed with $\mathrm{qS} \mathrm{cript}^{\mathrm{TM}} \mathrm{cDNA}$ superMix (Quanta Biosciences, Gaithersburg, Maryland, USA).

Real-time PCR was performed on ViiA ${ }^{\mathrm{TM}} 7$ with TaqMan custom-designed arrays for expression in both the circulating whole blood and PBMCs (Applied Biosystems, Foster City, CA, USA). The gene expression assays for TNF- $\alpha$, IL- $1 \beta$, IL18, MCP-1, CX3CL1, and CX3CR1 were Hs01113624_g1, Hs01555410_m1, Hs01038788_m1, Hs00234140_m1, Hs 00171086_m1, and Hs 01922583_s1, respectively. All formats used $\beta 2$-microglobulin (HS99999907_m1) as endogenous 
TABLE 2: Glucometabolic variables according to the randomized groups at baseline and after 3 months. Median (25, 75 percentiles) is given.

\begin{tabular}{|c|c|c|c|c|c|}
\hline \multirow{2}{*}{ Parameter } & \multicolumn{2}{|c|}{ Baseline } & \multicolumn{2}{|c|}{3 months } & \multirow{2}{*}{$\Delta p$} \\
\hline & Saxagliptin $(n=6)$ & Placebo $(n=6)$ & Saxagliptin $(n=5)$ & Placebo $(n=6)$ & \\
\hline HbA1c (\%) & $7.5(7.1,8.3)$ & $7.3(6.8,7.7)$ & $6.9(6.7,7.4)^{*}$ & $7.2(6.9,7.7)$ & 0.017 \\
\hline Glucose (mmol/L) & $8.2(7.7,9.7)$ & $8.5(7.4,10.6)$ & $7.2(6.5,10.0)$ & $8.5(7.7,10.4)$ & 0.329 \\
\hline Insulin (pmol/L) & $72(55,139)$ & $60(43,104)$ & $58(52,116)$ & $71(38,119)$ & 0.818 \\
\hline C-peptide (pmol/L) & $1090(810,1573)$ & $1025(767,1345)$ & $897(758,1599)$ & $887(744,1649)$ & 0.818 \\
\hline
\end{tabular}

$*$ refers to intragroup change in the saxagliptin group, $p=0.042$; $\Delta p$ refers to difference in changes from baseline to 3 months between the groups.

control and TaqMan Universal PCR Master Mix (P/N 4324018). mRNA levels were determined by relative quantification (RQ) using the $\Delta \Delta \mathrm{CT}$ method [13].

Levels of biomarkers in the PBMC supernatants were assessed by customized magnetic Luminex Screening Assays multiplex kit (R\&D) with the Bio-plex ${ }^{\circledR}$ luminex $\mathrm{xMAP}^{\mathrm{TM}}$ technology. Circulating markers were determined by commercially available enzyme-linked immunosorbent assay (ELISA) kits from R\&D Systems (Abingdon, Oxon, UK).

2.4. Statistics. Calculations were performed using SPSS version 22.0 (SPSS Inc., USA). $p$ values $<0.05$ were defined as statistically significant. Data are given as proportions or median (25, 75 percentiles). Nonparametric statistics were used due to small sample size and several skewed variables. Differences between groups and differences in changes between the groups were analyzed by Mann-Whitney $U$ test. Fisher's exact 2-sided test was used for categorical variables. Within group changes were calculated with Wilcoxon signed-ranks test.

\section{Results}

Baseline characteristics of the patients, including use of medications, are given in Table 1. One patient in the saxagliptin group was excluded from analyses at the last visit due to elevated C-reactive protein (CRP) (>10 mg/L).

Two patients in the saxagliptin group experienced adverse events during the study period-one patient had urinary tract infection and another an episode of mild hypoglycemia.

HbA1c was significantly reduced in the saxagliptintreated group $(p=0.042)$, different from that in the placebo group $(p=0.017)$ (Table 2$)$. No other significant changes or differences in changes were observed between the groups for other glucometabolic variables (Table 2).

3.1. Gene Expression in Isolated PBMCs. The mean distribution of monocytes and lymphocytes in the mononuclear cells from all experiments was $9.6 \%$ and $90.4 \%$, respectively.

Levels of gene expression of the inflammatory markers in unstimulated and LPS-stimulated cells are shown in Table 3. Expected increase in LPS-stimulated cells were seen, except for MCP-1, which might be due to the short incubation time [14], and also for CX3CR1.

In unstimulated cells, we observed a significant increase in IL-10 expression from baseline in the saxagliptin-treated patients $(p=0.043)$, significantly different from that in the placebo $(p=0.009)$, however not in LPS-stimulated cells.
No differences in changes between the groups in the expression of the proinflammatory markers were observed, neither in unstimulated nor in LPS-stimulated cells (Table 3, Figures 1(a) and 1(b)).

3.2. Supernatant from Isolated PBMCs. No between group differences or differences in changes of the selected markers were recorded in supernatants from neither unstimulated nor LPS-stimulated cells (data not shown). IL-18 was not detectable in unstimulated cells with the assay used.

3.3. Gene Expression in Circulating Leukocytes. There were no significant differences between the groups in gene expression of the selected markers at baseline. IL-10 mRNA increased significantly after 3 months in the saxagliptin group $(p=0.043)$, significantly different from that in the controls $(p=0.032)$ (Figure $1(c)$ ). There was an intragroup reduction in CX3CR1 expression in the placebo group ( $p=0.043)$, however not significantly different from the change in the saxagliptin group $(p=0.151)$ (Figure 2).

3.4. Circulating Levels. There were no statistically significant differences in the circulating levels between the groups at baseline. IL-18 levels increased numerically in both groups during the intervention period, however, only significant in the saxagliptin treatment group $(p=0.043)$ (different from placebo, $p=0.009$ ) (Table 4$)$. IL-10 levels were all below detection limit of our assay.

\section{Discussion}

In a recent review, attempting to compare different DPP-4 inhibitor effects on pro- and antiatherogenic factors, only few studies on saxagliptin have been performed [15].

Saxagliptin treatment did not reduce the LPS induced proinflammatory response in PBMCs in our study. We could also not demonstrate any downregulation of proinflammatory genes or reduced proinflammatory circulating markers related to saxagliptin treatment. However, in the present study, an increase in IL-10 mRNA expression in isolated PBMCs was demonstrated, which is in line with previous results on sitagliptin [10]. The increase in IL-10 expression shown in circulating leukocytes strengthens our result. From the literature, IL-10 is primarily produced from subsets of T cells, macrophages, and dendritic cells [16]. However, which cell types that are involved in the upregulation seen in our study can only be speculated on, but lymphocytes, which are dominantly present in PBMCs and also highly present 


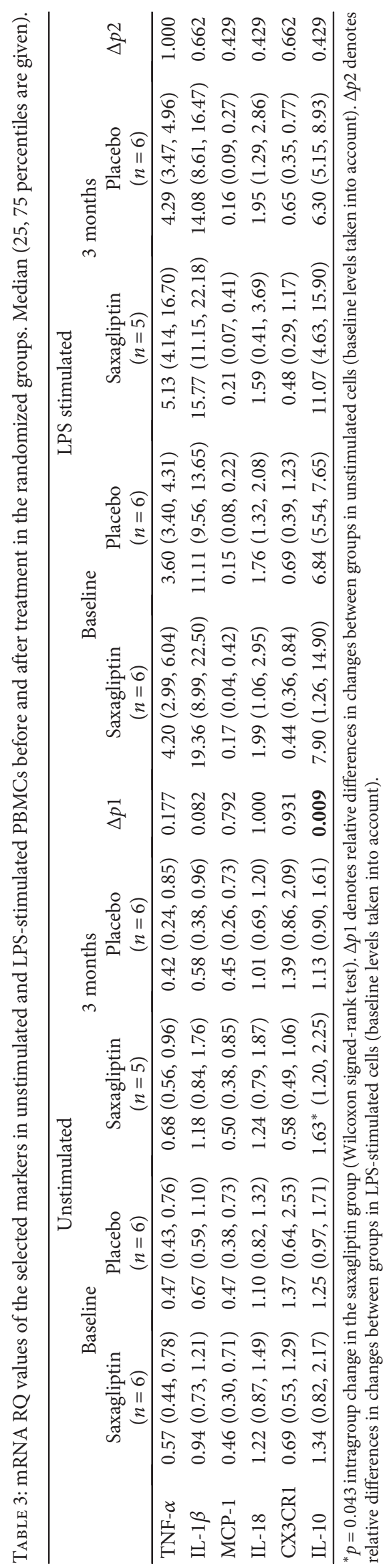




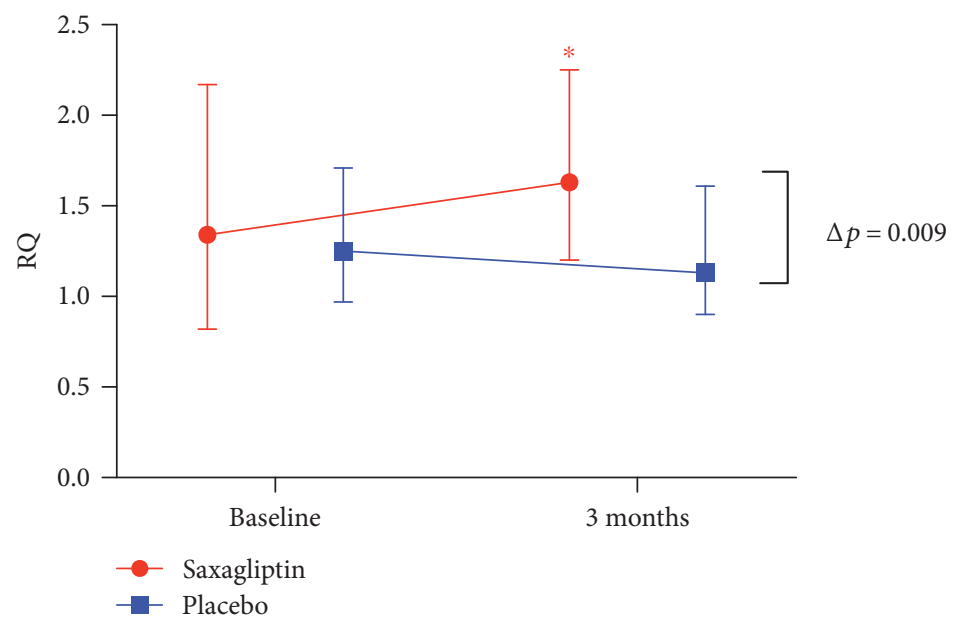

(a) Unstimulated PBMCs

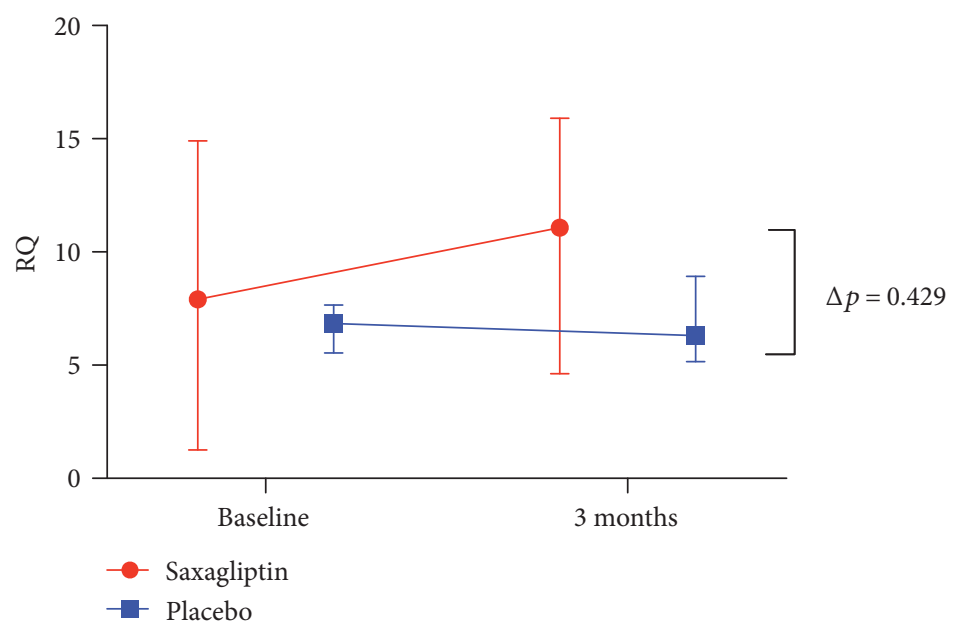

(b) LPS-stimulated PBMCs

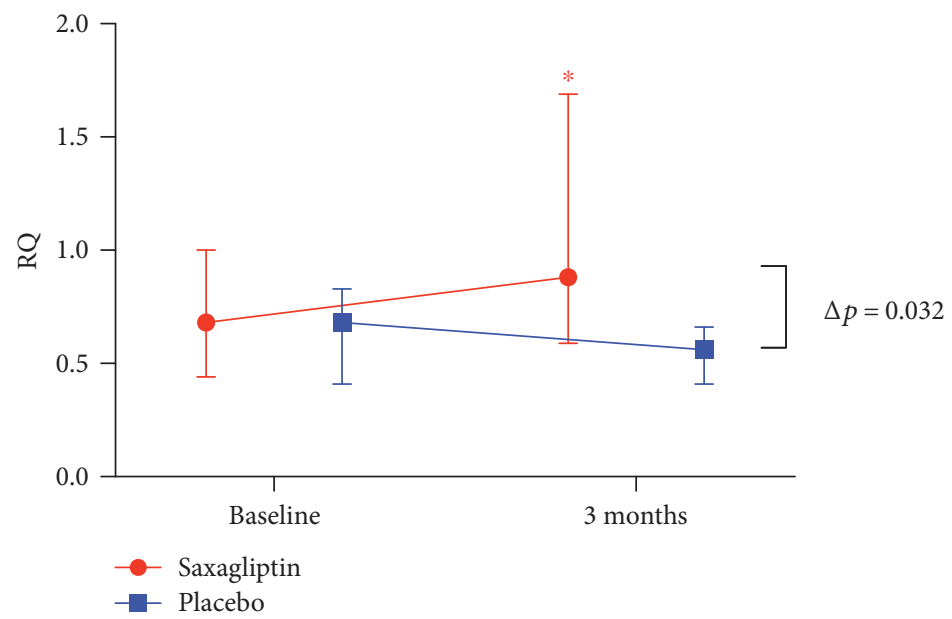

(c) Circulating leukocytes

FIgURE 1: The figure shows RQ values of IL-10 from (a) unstimulated PBMCs, (b) LPS-stimulated PBMCs, and (c) circulating leukocytes at baseline and after 3 months in the two randomized groups. The median values are given, with 25 and 75 percentiles depicting the spread. $\Delta p$ denotes relative differences in change between the groups. * depicts significant intragroup change in RQ value. $p<0.05$ for intragroup difference from baseline to 3 months. 

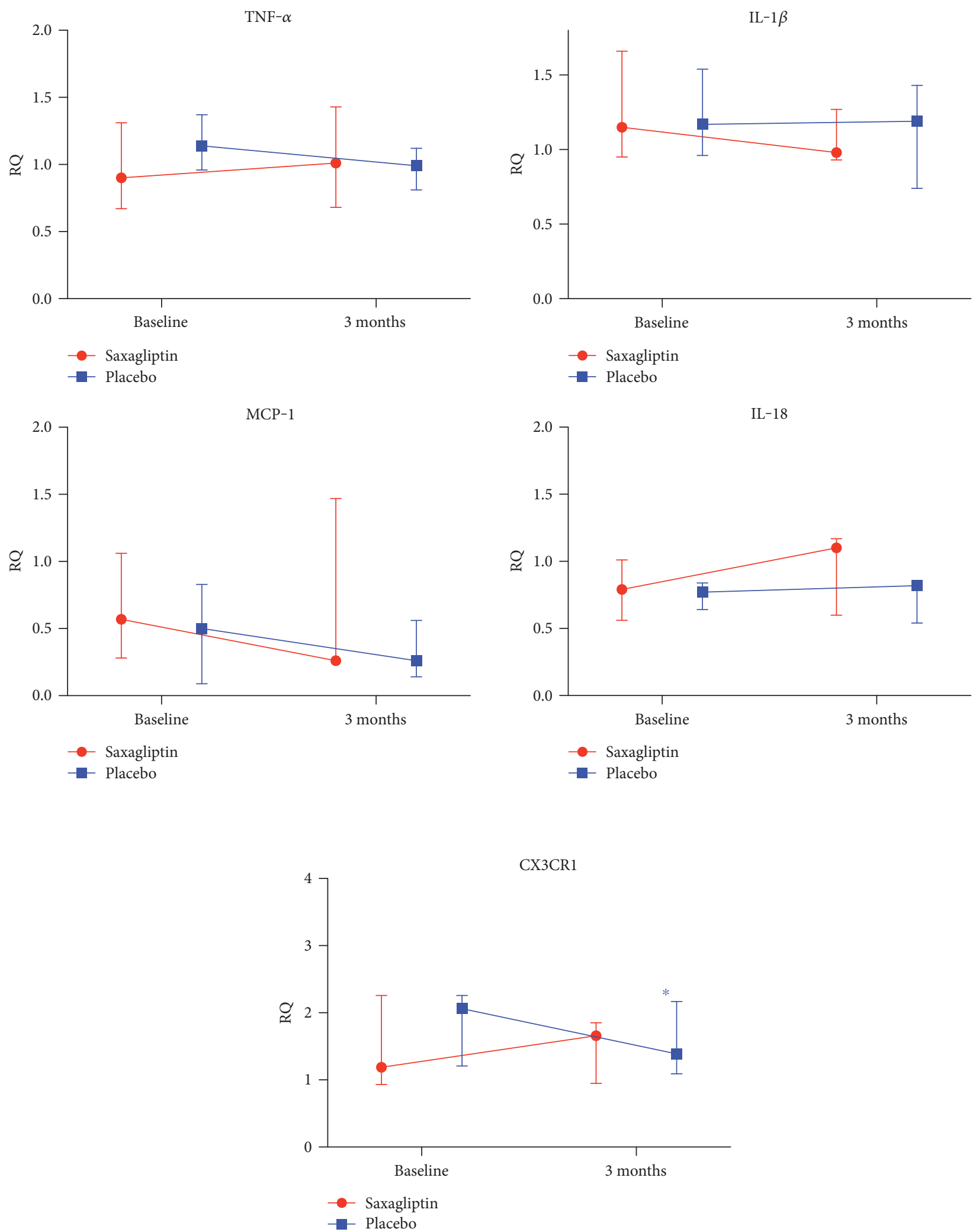

FIgURE 2: mRNA RQ values in circulating leukocytes before (baseline) and after treatment ( 3 months). Median values are given, with 25 and 75 percentiles depicting the spread. * Intragroup change, $p=0.043$. There were no statistically significant differences in relative changes of the selected proinflammatory markers between the groups. 
TABLE 4: Circulating levels of the selected markers before and after treatment in the randomized groups. Median (25, 75 percentiles) are given.

\begin{tabular}{|c|c|c|c|c|c|}
\hline \multirow{2}{*}{ Marker } & \multicolumn{2}{|c|}{ Baseline } & \multicolumn{3}{|c|}{3 months } \\
\hline & Saxagliptin $(n=6)$ & Placebo $(n=6)$ & Saxagliptin $(n=5)$ & Placebo $(n=6)$ & $\Delta p$ \\
\hline TNF- $\alpha(\mathrm{pg} / \mathrm{mL})$ & $1.61(1.36,1.96)$ & $1.23(1.04,1.49)$ & $1.54(1.32,1.64)$ & $1.33(1.16,1.59)$ & 0.429 \\
\hline MCP-1 (pg/mL) & $117(100,149)$ & $118(94,173)$ & $114(99,116)$ & $113(88,166)$ & 0.792 \\
\hline IL-18 (pg/mL) & $297(192,493)$ & $399(307,500)$ & $342(177,422)^{*}$ & $419(305,522)$ & 0.009 \\
\hline Fractalkine $(\mathrm{pg} / \mathrm{mL})$ & $604(535,649)$ & $520(455,628)$ & $577(451,627)$ & $504(443,622)$ & 0.931 \\
\hline
\end{tabular}

$\Delta p$ denotes difference in relative change between groups (Mann-Whitney $U$ test); ${ }^{*} p=0.043$ for intragroup change (Wilcoxon signed-rank test).

among circulating cells, might be the main source. We could, however, not find a corresponding increase in supernatants of the cell cultures, which might be due to the experiment time frame [17], and levels of IL-10 in the circulation were also not detectable, although a high sensitivity assay was used.

The increase in IL-10 levels might be speculated upon as a possible anti-inflammatory effect of saxagliptin. However, it might also be related to the observed reduction in $\mathrm{HbA1c}$, which fits with studies showing a reduction of IL-10 in response to elevated glucose levels $[18,19]$.

We observed an increase in circulating IL-18 in the patients treated with saxagliptin. This is in contrast to another study reporting a significant reduction in IL-18 with gliptins [20]. This report was, however, not from patients with cardiovascular disease, and two other DPP-4 inhibitors were used. It should, nevertheless, be emphasized that the number of patients was low and the values highly spread in our study, and the results should therefore be interpreted with caution. In another study, it was reported on no effect of long-term treatment ( 24 months) with alogliptin on other selected inflammatory markers [11].

All patients included in our study were using medications also for their CAD; thus, all were using statins and many antiplatelet therapies. Therefore, a possible antiinflammatory effect might be masked by these drugs, which also have anti-inflammatory effects $[21,22]$. The use of medications in general in such populations, may complicate the reliability of the results. Nevertheless, this is the normal situation in such patients and new antidiabetic drugs might add to the positive effects of other drugs.

The large SAVOR-TIMI 53 study showed that adding saxagliptin to standard of care did not increase nor decrease cardiovascular ischemic events in high-risk patients. But they did, however, show a $27 \%$ greater risk of hospitalization for heart failure [23]. Also, other clinical trials have been neutral with regard to DPP-4 inhibitor treatment on cardiovascular event rates $[24,25]$. The conclusion so far has been that the investigated DPP-4 inhibitors have been noninferior for cardiovascular safety, however not demonstrating superiority, meaning beneficial effects of DPP-4 inhibition on cardiovascular risk [26]. A new drug, empagliflozin (sodium-glucose cotransporter 2 (SGLT2) inhibitor), has recently gained a lot of interest since it seems to reduce cardiovascular mortality compared to placebo [27].

The main limitation of our study is the low number of patients included. Thus, this is a small, purely hypothesis generating study, with potential type 2 statistical error, and our results need to be discussed and interpreted with caution.
Nevertheless, for the ex vivo model of isolated PBMCs, the number might be considered satisfactory. We also obtained expected reductions in $\mathrm{HbAlc}$ levels in the saxagliptin group, indicative of good compliance to the study drug.

\section{Conclusion}

Taken together, saxagliptin treatment in patients with combined CAD and T2DM for 3 months did not downregulate proinflammatory markers in neither isolated PBMCs nor circulating leukocytes, whereas the anti-inflammatory cytokine IL-10 was upregulated in both models. A possible antiinflammatory effect of saxagliptin, as observed in the present study with increased expression of IL-10, needs to be confirmed in larger studies.
Abbreviations
CAD: Coronary artery disease
CRP: C-reactive protein
CX3CL1: Fractalkine
CX3CR1: Fractalkine receptor
DPP-4: Dipeptidyl peptidase 4
IL: Interleukin
LPS: $\quad$ Lipopolysaccharide
MCP-1: Monocyte chemoattractant protein-1
PBMCs: Peripheral blood mononuclear cells
T2DM: $\quad$ Type 2 diabetes
TNF- $\alpha$ : Tumor necrosis factor alpha.

\section{Conflicts of Interest}

The authors declare no competing interests.

\section{Authors' Contributions}

Ida Unhammer Njerve was involved in the planning of the study, recruitment and follow-up of patients, data analyses and interpretation, and drafting and finalization the manuscript. Sissel Åkra, Reidun Øvstebø, and Hans Christian D. Aass were involved in the laboratory analyses and data interpretation. Thomas W. Weiss was involved in planning the study and the discussion of the manuscript. Rune Byrkjeland was involved in the recruitment and follow-up of study patients. Svein Solheim, Harald Arnesen, and Ingebjørg Seljeflot contributed to the planning of the study, data interpretation, and drafting and discussion of the manuscript. All authors have read and approved the manuscript 
before submission. Ida Unhammer Njerve and Sissel Åkra have equal contributions (shared first authorship).

\section{Acknowledgments}

The authors thank Trine B. Opstad, Vibeke Bratseth, and Beate Vestad for their excellent laboratory assistance. Bristol-Myers Squibb Ltd. provided tablets of saxagliptin $5 \mathrm{mg}$ and placebo without any scientific restrictions. The study was followed up by AstraZeneca after the divestiture in Feb. 2014. The study was further supported by the Stein Erik Hagens Foundation for Clinical Heart Research, Oslo, Norway.

\section{References}

[1] S. Dhillon, "Saxagliptin: a review in type 2 diabetes," Drugs, vol. 75, no. 15, pp. 1783-1796, 2015.

[2] S. E. Inzucchi, R. M. Bergenstal, J. B. Buse et al., "Management of hyperglycaemia in type 2 diabetes, 2015: a patient-centred approach. Update to a position statement of the American Diabetes Association and the European Association for the study of diabetes," Diabetologia, vol. 58, no. 3, pp. 429-442, 2015.

[3] D. J. Drucker and M. A. Nauck, "The incretin system: glucagon-like peptide-1 receptor agonists and dipeptidyl peptidase- 4 inhibitors in type 2 diabetes," Lancet, vol. 368, no. 9548, pp. 1696-1705, 2006.

[4] G. P. Fadini and A. Avogaro, "Cardiovascular effects of DPP-4 inhibition: beyond GLP-1," Vascular Pharmacology, vol. 55, no. 1-3, pp. 10-16, 2011.

[5] F. Vittone, A. Liberman, D. Vasic et al., "Sitagliptin reduces plaque macrophage content and stabilises arteriosclerotic lesions in Apoe (-/-) mice," Diabetologia, vol. 55, no. 8, pp. 2267-2275, 2012.

[6] J. Matsubara, S. Sugiyama, K. Sugamura et al., "A dipeptidyl peptidase-4 inhibitor, des-fluoro-sitagliptin, improves endothelial function and reduces atherosclerotic lesion formation in apolipoprotein E-deficient mice," Journal of the American College of Cardiology, vol. 59, no. 3, pp. 265-276, 2012.

[7] Z. Shah, T. Kampfrath, J. A. Deiuliis et al., "Long-term dipeptidyl-peptidase 4 inhibition reduces atherosclerosis and inflammation via effects on monocyte recruitment and chemotaxis," Circulation, vol. 124, no. 21, pp. 2338-2349, 2011.

[8] J. Matsubara, S. Sugiyama, E. Akiyama et al., "Dipeptidyl peptidase- 4 inhibitor, sitagliptin, improves endothelial dysfunction in association with its anti-inflammatory effects in patients with coronary artery disease and uncontrolled diabetes," Circulation Journal, vol. 77, no. 5, pp. 1337-1344, 2013.

[9] A. Makdissi, H. Ghanim, M. Vora et al., "Sitagliptin exerts an antinflammatory action," The Journal of Clinical Endocrinology and Metabolism, vol. 97, no. 9, pp. 3333-3341, 2012.

[10] N. Satoh-Asahara, Y. Sasaki, H. Wada et al., "A dipeptidyl peptidase-4 inhibitor, sitagliptin, exerts anti-inflammatory effects in type 2 diabetic patients," Metabolism, vol. 62, no. 3, pp. 347-351, 2013.

[11] T. Mita, N. Katakami, H. Yoshii et al., "Alogliptin, a dipeptidyl peptidase 4 inhibitor, prevents the progression of carotid atherosclerosis in patients with type 2 diabetes: the Study of Preventive Effects of Alogliptin on Diabetic Atherosclerosis (SPEAD-A)," Diabetes Care, vol. 39, no. 1, pp. 139-148, 2016.
[12] R. Øvstebø, H. C. Aass, K. B. Haug et al., "LPS from Neisseria meningitidis is crucial for inducing monocyte- and microparticle-associated tissue factor activity but not for tissue factor expression," Innate Immunity, vol. 18, no. 4, pp. 580591, 2012.

[13] K. J. Livak and T. D. Schmittgen, "Analysis of relative gene expression data using real-time quantitative PCR and the $2(-\Delta \Delta \mathrm{C}(\mathrm{T}))$ method," Methods, vol. 25, no. 4, pp. 402-408, 2001.

[14] A. S. Møller, R. Øvstebø, A. B. Westvik, G. B. Joø, K. B. Haug, and P. Kierulf, "Effects of bacterial cell wall components (PAMPs) on the expression of monocyte chemoattractant protein-1 (MCP-1), macrophage inflammatory protein- $1 \alpha$ $(\mathrm{MIP}-1 \alpha)$ and the chemokine receptor CCR2 by purified human blood monocytes," Journal of Endotoxin Research, vol. 9, no. 6, pp. 349-360, 2003.

[15] T. P. Singh, V. N. Vangaveti, and U. H. Malabu, "Dipeptidyl peptidase- 4 inhibitors and their potential role in the management of atherosclerosis-a review," Diabetes and Metabolic Syndrome: Clinical Research and Reviews, vol. 9, no. 4, pp. 223-229, 2015.

[16] K. N. Couper, D. G. Blount, and E. M. Riley, "IL-10: The master regulator of immunity to infection," Journal of Immunology, vol. 180, no. 9, pp. 5771-5777, 2008.

[17] M. R. de Waal, J. Abrams, B. Bennett, C. G. Figdor, and J. E. de Vries, "Interleukin 10 (IL-10) inhibits cytokine synthesis by human monocytes: an autoregulatory role of IL-10 produced by monocytes," The Journal of Experimental Medicine, vol. 174, no. 5, pp. 1209-1220, 1991.

[18] D. Reinhold, S. Ansorge, and E. D. Schleicher, "Elevated glucose levels stimulate transforming growth factor- $\beta 1$ (TGF- $\beta 1$ ), suppress interleukin IL-2, IL-6 and IL-10 production and DNA synthesis in peripheral blood mononuclear cells," Hormone and Metabolic Research, vol. 28, no. 6, pp. 267-270, 1996.

[19] I. Torres-Castro, Ú. D. Arroyo-Camarena, C. P. MartínezReyes et al., "Human monocytes and macrophages undergo M1-type inflammatory polarization in response to high levels of glucose," Immunology Letters, vol. 176, pp. 81-89, 2016.

[20] M. R. Rizzo, M. Barbieri, R. Marfella, and G. Paolisso, "Reduction of oxidative stress and inflammation by blunting daily acute glucose fluctuations in patients with type 2 diabetes: role of dipeptidyl peptidase-IV inhibition," Diabetes Care, vol. 35, no. 10, pp. 2076-2082, 2012.

[21] M. Takemoto and J. K. Liao, "Pleiotropic effects of 3-hydroxy3-methylglutaryl coenzyme a reductase inhibitors," Arteriosclerosis, Thrombosis, and Vascular Biology, vol. 21, no. 11, pp. 1712-1719, 2001.

[22] K. A. Müller, M. Chatterjee, D. Rath, and T. Geisler, "Platelets, inflammation and anti-inflammatory effects of antiplatelet drugs in ACS and CAD," Thrombosis and Haemostasis, vol. 114, no. 3, pp. 498-518, 2015.

[23] B. M. Scirica, D. L. Bhatt, E. Braunwald et al., "Saxagliptin and cardiovascular outcomes in patients with type 2 diabetes mellitus," The New England Journal of Medicine, vol. 369, no. 14, pp. 1317-1326, 2013.

[24] J. B. Green, M. A. Bethel, P. W. Armstrong et al., "Effect of sitagliptin on cardiovascular outcomes in type 2 diabetes," The New England Journal of Medicine, vol. 373, no. 3, pp. 232242, 2015.

[25] W. B. White, C. P. Cannon, S. R. Heller et al., "Alogliptin after acute coronary syndrome in patients with type 2 diabetes," The 
New England Journal of Medicine, vol. 369, no. 14, pp. 13271335, 2013.

[26] T. V. Fiorentino and G. Sesti, "Lessons learned from cardiovascular outcome clinical trials with dipeptidyl peptidase 4 (DPP4) inhibitors," Endocrine, vol. 53, no. 2, pp. 372-380, 2016.

[27] B. Zinman, C. Wanner, J. M. Lachin et al., "Empagliflozin, cardiovascular outcomes, and mortality in type 2 diabetes," The New England Journal of Medicine, vol. 373, no. 22, pp. 21172128, 2015. 


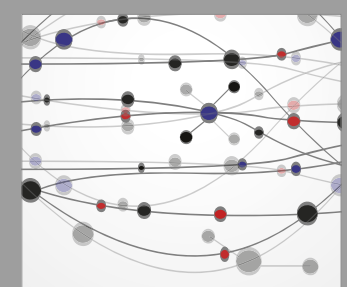

The Scientific World Journal
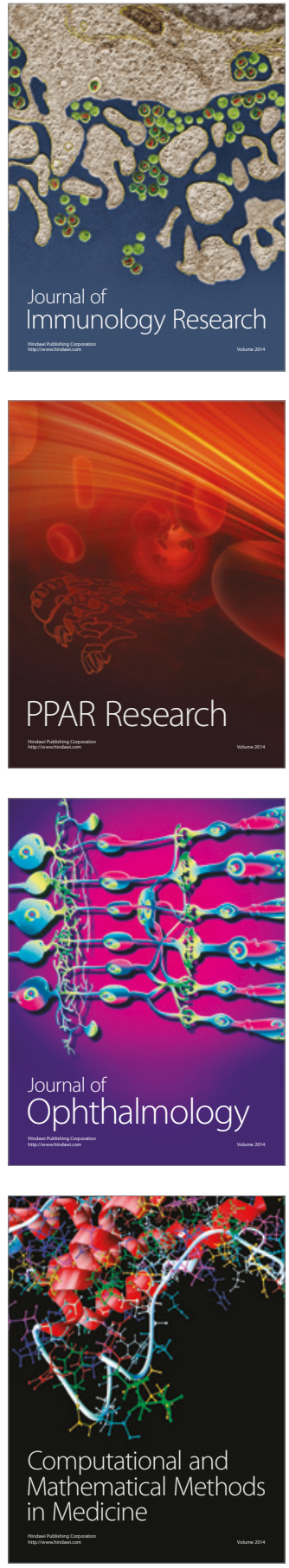

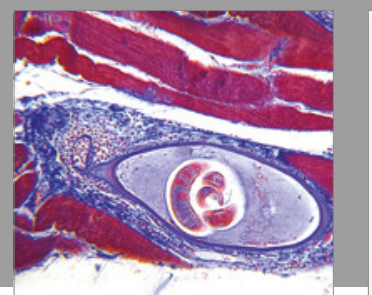

Gastroenterology Research and Practice
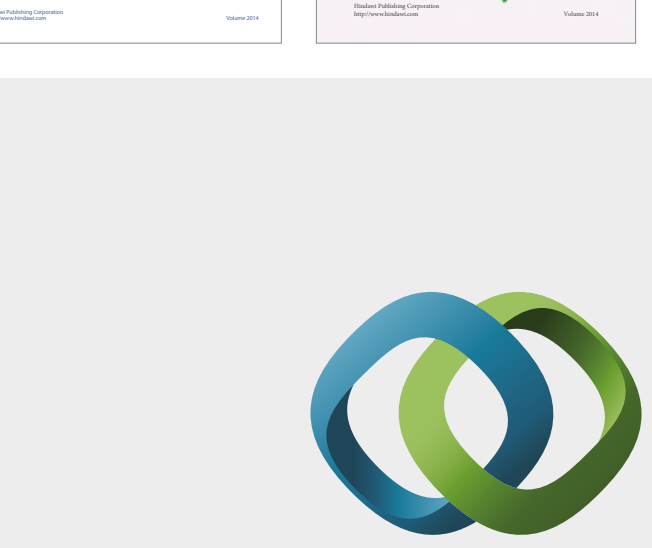

\section{Hindawi}

Submit your manuscripts at

https://www.hindawi.com
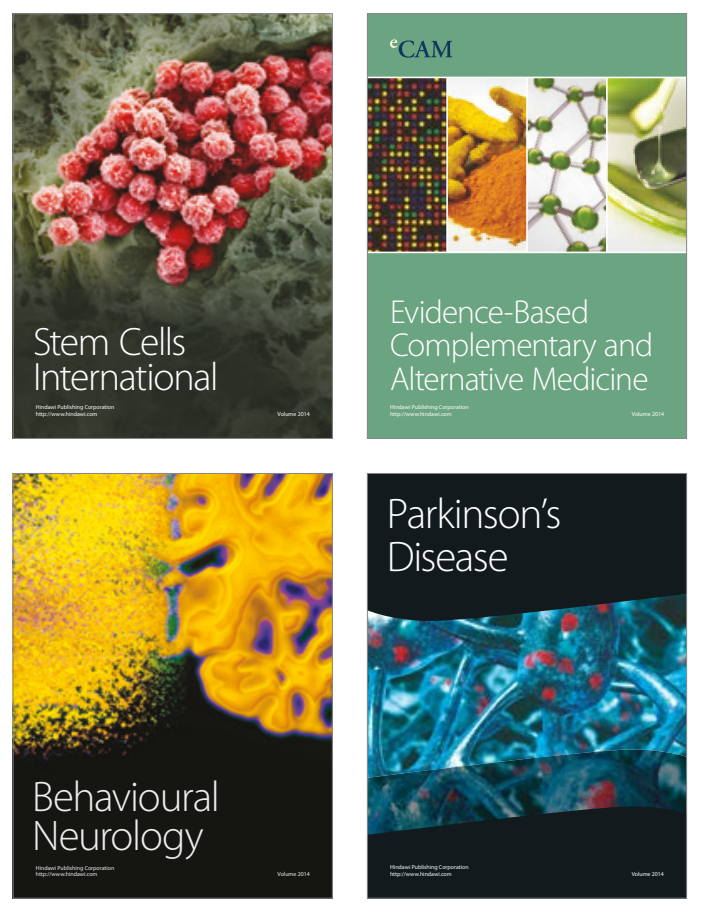
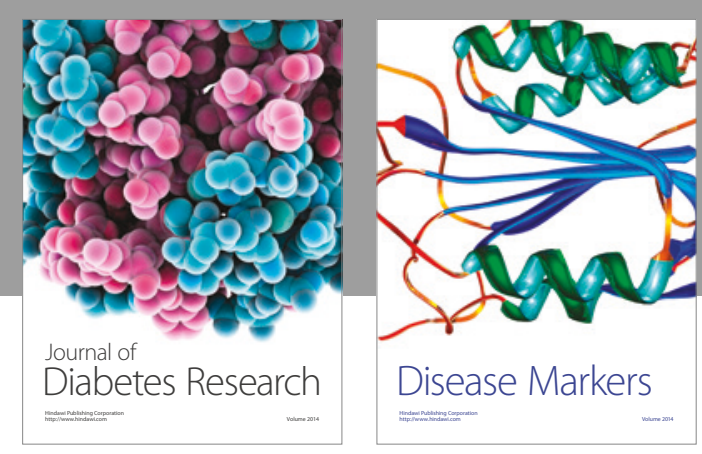

Disease Markers
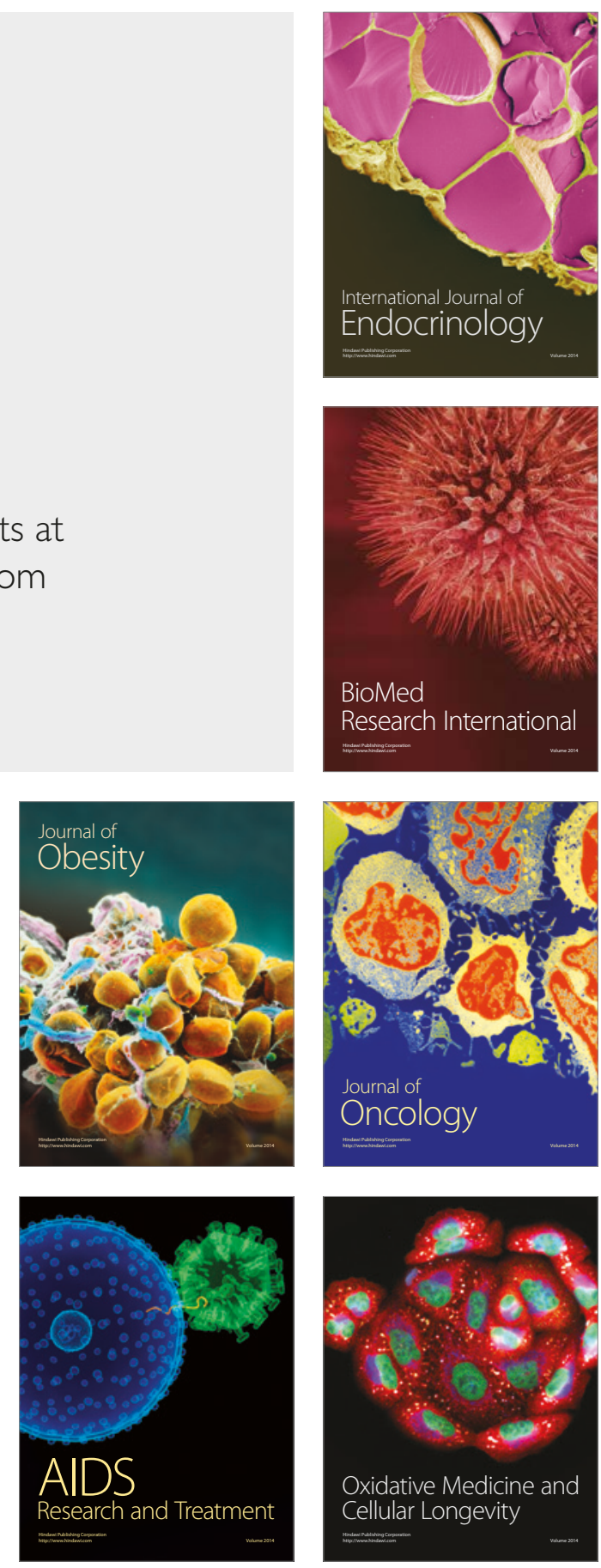